\title{
Observation of Edge Transport in the Disordered Regime of Topologically Insulating InAs/GaSb Quantum Wells
}

\author{
Ivan Knez, Charles T. Rettner, See-Hun Yang, and Stuart S. P. Parkin \\ IBM Research-Almaden, San Jose, California 95120, USA \\ Lingjie Du, and Rui-Rui Du \\ Department of Physics and Astronomy, Rice University, Houston, Texas 77251-1892, USA \\ Gerard Sullivan \\ Teledyne Scientific and Imaging, Thousand Oaks, California 91630, USA \\ (Received 7 October 2013; published 15 January 2014)
}

\begin{abstract}
We observe edge transport in the topologically insulating InAs/GaSb system in the disordered regime. Using asymmetric current paths we show that conduction occurs exclusively along the device edge, exhibiting a large Hall signal at zero magnetic fields, while for symmetric current paths, the conductance between the two mesoscopicly separated probes is quantized to $2 e^{2} / h$. Both quantized and self-averaged transport show resilience to magnetic fields, and are temperature independent for temperatures between $20 \mathrm{mK}$ and $1 \mathrm{~K}$.
\end{abstract}

DOI: 10.1103/PhysRevLett.112.026602

PACS numbers: 72.25.Dc, 73.23.-b, 73.63.Hs

Two-dimensional (2D) topological insulators (TI) are a novel class of materials that are insulating in the bulk but which display uniquely conductive edge channels [1-4]. These one-dimensional (1D) edge modes are helical, with the spin direction tied to the electron direction of motion, and are protected from backscattering by the time reversal symmetry (TRS) [5,6]. Applying magnetic fields breaks the TRS, removing the topological protection of the $1 \mathrm{D}$ helical liquid (HL) from single particle backscattering, resulting in a gap opening in the edge spectrum. Such HL channels were first observed in transport measurements in $\mathrm{HgTe} / \mathrm{CdTe}$ quantum wells, and much of the HL phenomenology has been confirmed and elucidated in those first experiments [7,8]. Recently, Du et al. reported quantized transport in the inverted regime of Si-doped InAs/GaSb quantum wells in mesoscopic samples [9], where the existence of helical edge states was proposed in Ref. [10] and the first experimental evidence provided in Ref. [11] through scaling arguments due to the presence of residual bulk carriers. Unlike that observed in $\mathrm{HgTe} / \mathrm{CdTe}$ [7], quantized transport in InAs/GaSb persists to magnetic fields of several Tesla $[9,11]$, challenging the common understanding of 2D TIs in terms of TRS protected edge states and associated $Z_{2}$ topological invariant.

Much remains to be learned about the nature and robustness of $\mathrm{HL}$, in particular, to TRS breaking and disorder. In addition, edge transport in InAs/GaSb has so far only been indirectly assessed in ballistic samples $[9,11]$. In this Letter we study TI InAs/GaSb quantum wells in the disordered regime [12], where the total device size is much larger than the ballistic length of the HL, and show that transport in the topological regime manifestly occurs along the sample perimeter and is quantized to values consistent with the existence of a HL. Similar to ballistic regime studies $[9,11]$, the conduction is also seen to be only weakly dependent on externally applied magnetic fields of up to $1 \mathrm{~T}$. We argue that this behavior is due to the reduced effective $g$ factor of the edge states originating from their small Fermi velocity $v_{F}$. In addition, the edge states do not exhibit significant variation in transport properties for temperatures between $20 \mathrm{mK}$ and $1 \mathrm{~K}$ measured. This is in contrast to theoretical studies, which have predicted power law corrections to the edge conductance as a function of temperature due to single particle inelastic [13-15], and correlated two-particle backscattering [16], while such corrections vanish for Kondo scattering from a magnetic impurity in a dc limit [17]. The absence of such power laws in the temperature dependence of the edge conductance observed here indicates that spinflip single particle backscattering $[14,18]$ is the dominant edge scattering process in this system.

Two contacts to the HL are connected by a quantum of resistance $h / e^{2}$, in the ballistic limit, where the contact separation $L$ is smaller than the characteristic length $L_{\mathrm{ch}}$, determined by the backscattering processes. Furthermore, in this limit, the edge transport exhibits universal conductance fluctuations as the Fermi energy is tuned through the topological bulk gap [7]. On the other hand, in the opposite limit of the disordered regime where $L \gg L_{\mathrm{ch}}$, two contacts to the helical edge are connected by a series of quantum resistors with a total resistance value of $R=\left(h / e^{2}\right)\left(L / L_{\mathrm{ch}}\right) \cdot[11,18]$ In addition, conductance fluctuations average out, so that the edge resistance in this limit is referred to as a "self-averaged resistance" [15]. The 
length dependence property of self-averaged helical edge resistance is here used to asymmetrically inject currents into the left and right edges of the device, which are of unequal length, thus generating a large Hall voltage at zero magnetic fields and directly showing that the conduction, indeed, occurs only along the boundary of the system. On the other hand, when the left and right edges of the device are of equal length, the currents injected into the left and right leads are approximately the same, and equal to half of the total injected current $I$. In this case, the Hall voltage vanishes, and the voltage drop between two ballistic probes on one edge of the device is quantized to $V=\left(h / e^{2}\right)(I / 2)$.

Measurements are performed on the samples from the same wafer as reported in Ref. [9], with 125 A InAs and $100 \mathrm{~A} \mathrm{GaSb}$ double quantum wells in the inverted regime, hosting electron and hole 2D layers, respectively. The wells are Si doped in order to suppress the bulk conductivity, arising from reduced hybridization of electron hole states due to disorder $[19,20]$. Samples are fabricated via standard lithography techniques, ion milling, and ion beam deposition of electrical contacts and $\mathrm{SiO}_{2}$ dielectric layer, which defines the front electrostatic gate. The doped $\mathrm{N}^{+}$GaAs substrate serves as a bottom electrostatic gate. Measurements are performed in a dilution refrigerator with a base temperature of $20 \mathrm{mK}$ and a vector magnet with magnetic fields up to $1 \mathrm{~T}$. Standard lock-in techniques with excitation currents as low as $1 \mathrm{nA}$ have been used.

The device is defined into a rectangular mesa $10 \mu \mathrm{m}$ in width and $40 \mu \mathrm{m}$ in length with several contacts as shown in the inset to Fig. 1(b). The system band structure, with colored spin-up and spin-down edge states inside the bulk gap, is shown in the inset of Fig. 1(c). First, we pass current from contact 1 while grounding contact 4 and measure the Hall voltage between leads 6 and 2 as a function of the front and back gating biases, $V_{\text {front }}$ and $V_{\text {back }}$, respectively, at zero magnetic field, $B=0 \mathrm{~T}$. The Hall resistance map $R_{1-4,6-2}$ as a function of gate biases is shown in Fig. 1(a), and shows large values of $R_{1-4,6-2} \sim-10 \mathrm{k} \Omega$ for gate voltages in the topological regime with the Fermi energy, $E_{F}$, in the bulk gap. In contrast, no Hall signal is detected for $E_{F}$ in the electron or hole bands, i.e., right and left white portions of the same figure, respectively. Entry into the topological bulk gap is verified via Hall measurements at finite magnetic fields $[11,20]$. The wave vector at which electron and hole bands from InAs and GaSb, respectively, cross is $k_{\text {cross }} \sim 1.67 \times 10^{6} \mathrm{~cm}^{-1}$, and shows little dependence on the back gate bias. On the other hand, when the grounding contact is switched from lead 4 to lead 3, the zero-field Hall resistance $R_{1-3,6-2}$ in the topological regime changes sign and is $R_{1-3,6-2} \sim 10 \mathrm{k} \Omega$ as shown in Fig. 1(b), while when both contacts 4 and 3 are grounded the Hall resistance $R_{1-3 \& 4,6-2}$ is suppressed [Fig 1(c)].

The large Hall voltage at $B=0$ T observed here directly shows that the current flows solely along the sample perimeter, and is a consequence of the asymmetry of the
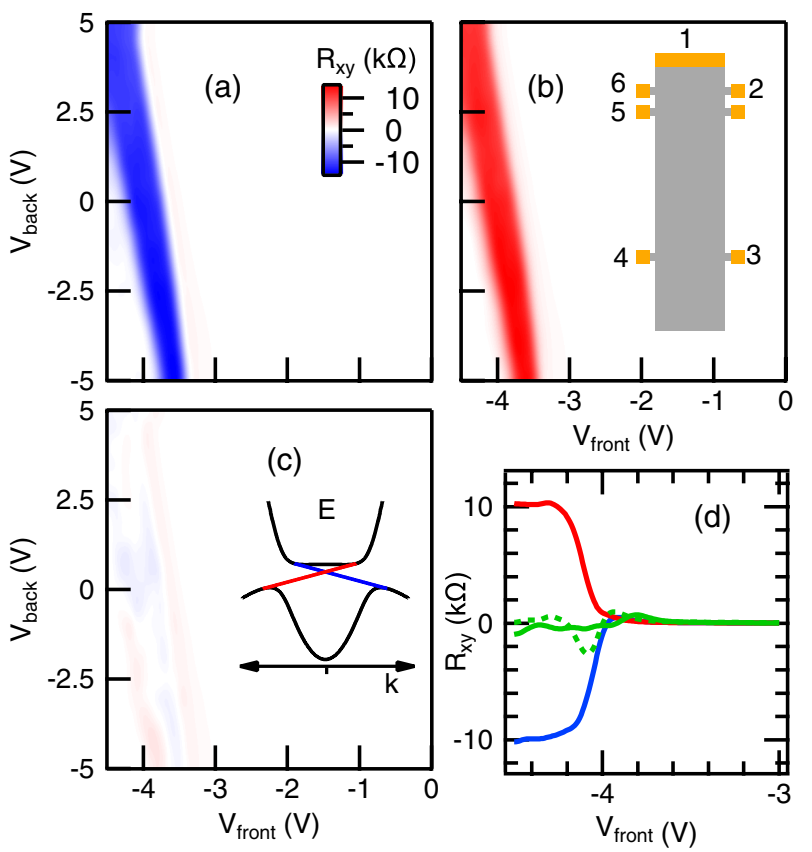

FIG. 1 (color online). Panels (a)-(c) show the Hall resistance $R_{1-4,6-2}, R_{1-3,6-2}$, and $R_{1-3 \& 4,6-2}$, respectively, as a function of front and back gate biases, $V_{\text {front }}$ and $V_{\text {back }}$, at zero magnetic fields, $B=0 \mathrm{~T}, T=20 \mathrm{mK}$. Inset of panel (b) shows the geometry of the device while the band structure with spin-up and spin-down edge states in red and blue, respectively, is shown in the inset of panel (c). Voltage is measured between leads 6 and 2 , and current is passed from lead 1, while lead 3, 4, 3 and 4, are grounded in panels (a)-(c), respectively. Large Hall resistance in panels (a) and (b) for gate biases in the bulk gap is a direct evidence of the edge transport and arises due to the length asymmetry of current paths along the left and right edges, while the Hall resistance is suppressed for symmetric paths in panel (c). Panel (d) shows $R_{1-4,6-2}, R_{1-3,6-2}$, and $R_{1-3 \& 4,6-2}$ in blue, red, and green, respectively, at fixed $V_{\text {back }}=5 \mathrm{~V}$, while $R_{1-4,6-2}+$ $R_{1-3,6-2}$ is given in the green dashed line for reference.

currents flowing in the left and right edge paths. These currents are given as $I_{\text {left }}=I\left(R_{\text {right }} / R_{\text {right }}+R_{\text {left }}\right)$ and $I_{\text {right }}=I\left(R_{\text {left }} / R_{\text {right }}+R_{\text {left }}\right)$, where $R_{\text {left }}$ and $R_{\text {right }}$ are self-averaged resistances of the left and right edge channels, respectively, and are directly proportional to corresponding edge lengths, while $I$ is the total current. In the case of the near ballistic separation of contacts 1 and 2 as well as 1 and 6, the Hall resistance detected in Figs. 1(a) and $1(\mathrm{~b})$ is $R_{1-4,6-2}=-R_{1-3,6-2}=\left(h / e^{2}\right)\left(I_{\text {right }}-I_{\text {left }}\right)=$ $\left(h / e^{2}\right)\left(R_{\text {left }}-R_{\text {right }} / R_{\text {right }}+R_{\text {left }}\right)$. The short path distance between contacts 1 and 4 on the left edge is the same as the distance between 1 and 3 on the right edge and equals $25 \mu \mathrm{m}$ while the long path distance between 1 and 4 ( 1 and 3 ), i.e., passing through contacts 1-2-3-4 (1-6-5-4) is $50 \mu \mathrm{m}$. As a result, we expect $R_{1-4,6-2}$ to be equal to $-R_{1-3,6-2} \sim-h / 3 e^{2}=-8.6 \mathrm{k} \Omega$. The actual separation between the contacts 1 and 2 as well as 1 and 6 is $L_{1-2}=L_{1-6}=2.9 \mu \mathrm{m}$, which is slightly larger than the 
ballistic length of the HL, as determined in the latter parts of this Letter, accounting for the slightly larger Hall resistance values observed in Figs. 1(a) and 1(b). In fact, noting that the edge resistance scales roughly linearly in regime when $L$ is slightly larger than $L_{\mathrm{ch}}$, then $R_{1-4,6-2}=$ $-R_{1-3,6-2} \sim-\left(L_{1-2} / L_{\mathrm{ch}}\right)\left(h / 3 e^{2}\right)$ and this nominal value is only one percent higher than the observed values shown in Fig. 1(d) for a fixed $V_{\text {back }}=5 \mathrm{~V}$. A lump circuit type analysis places a lower bound on the bulk shunting resistance to at least several $\mathrm{M} \Omega$. When the left and right edge current paths are of equal length then the Hall voltage will be suppressed, as observed in Fig. 1(c) for symmetrically grounded devices. The slight deviation of Hall resistance values in this case, is due to the differing scattering potentials along the left and right edges that vary with the gate bias, making the left and right paths electrically unequal.

Besides the large transverse signal, edge transport is also observed in measurements of the longitudinal resistance as shown in Fig. 2. In this case, the voltage is measured between contacts 6 and 5 , where $L_{6-5}=1.8 \mu \mathrm{m}$, and current is passed from lead 1 , while lead 3, 4, 3 and 4, are grounded in separate measurements. Resulting resistance maps, $R_{1-4,6-5}, R_{1-3,6-5}$, and $R_{1-3 \& 4,6-5}$ as a function
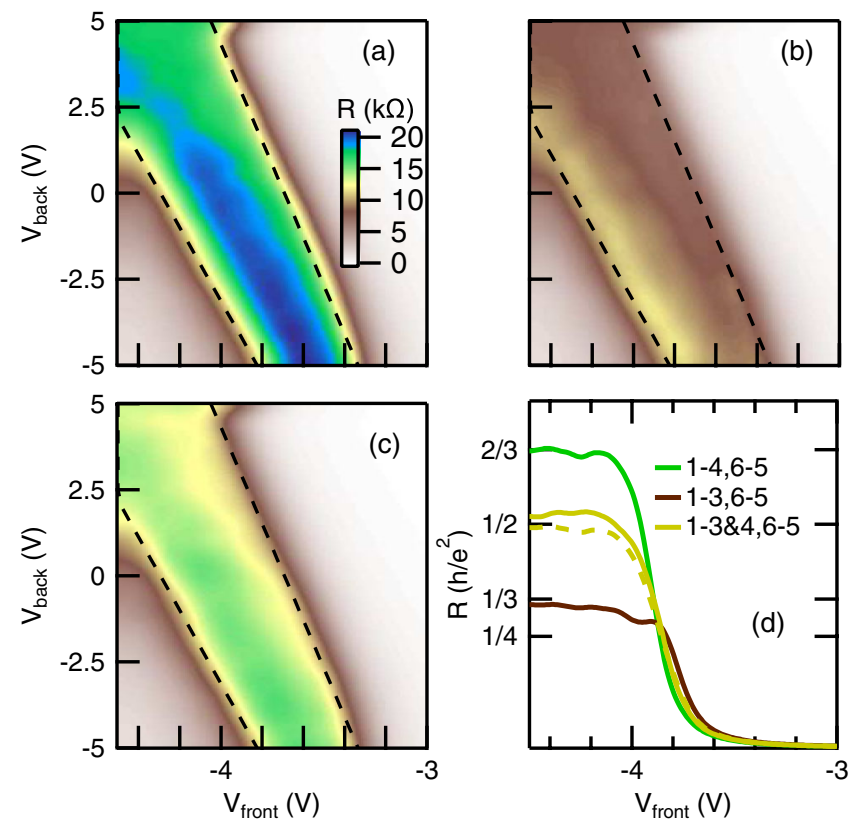

FIG. 2 (color online). Panels (a)-(c) show longitudinal resistance $R_{1-4,6-5}, R_{1-3,6-5}$, and $R_{1-3 \& 4,6-5}$, with $L_{6-5}=1.8 \mu \mathrm{m}$ in the ballistic regime, at $B=0 \mathrm{~T}, T=20 \mathrm{mK}$, as a function of $V_{\text {front }}$ and $V_{\text {back }}$, and for fixed $V_{\text {back }}=5 \mathrm{~V}$ in panel (d) in green, brown, and yellow, respectively, while $\left(R_{1-4,6-5}+R_{1-3,6-5}\right) / 2$ is shown as the dashed line. Asymmetry in the lengths of the left and right edge paths gives $R_{1-4,6-5}=\left(h / e^{2}\right)(2 / 3)=17.2 \mathrm{k} \Omega$ and $R_{1-3,6-5}=\left(h / e^{2}\right)(1 / 3)=8.6 \mathrm{k} \Omega$, in panels (a) and (b), respectively, while for symmetric grounding the quantized value of $R_{1-3 \& 4,6-5}=\left(h / e^{2}\right)(1 / 2)=12.9 \mathrm{k} \Omega$ is observed. of gate biases at $B=0 \mathrm{~T}$ are shown in Figs. 2(a), 2(b), and 2(c), respectively. Figure 2(d) shows the cut through the resistance map at fixed $V_{\text {back }}=5 \mathrm{~V}$, while the $V_{\text {front }}$ is varied from -3 to $-4.5 \mathrm{~V}$. Similar to the Hall signal analysis, asymmetry in the lengths of the left and right edge paths for the case when either contact 4 or contact 3 is grounded, will give $R_{1-4,6-5}=\left(h / e^{2}\right)(2 / 3)$ and $R_{1-3,6-5}=\left(h / e^{2}\right)(1 / 3)$, respectively, while when both 3 and 4 are grounded, lengths of the left and right edge paths are the same, and current in the left and right edge is roughly half of the total current, giving the quantized value of $R_{1-3 \& 4,6-5}=\left(h / e^{2}\right)(1 / 2)$. Latter values are within one percent of the observed values in Fig. 2(d), thus demonstrating that the edge transport in InAs/GaSb is indeed consistent with the existence of 1D HL. While evidence supporting the helical property of the edge states has been previously presented in Ref. [21], proof of the spinmomentum locking is yet to be experimentally demonstrated, and we note that the presence of a spinless 1D edge channel would also account for the observed transport behavior. However, a lack of topological protection from backscattering would likely localize such states on the tens of micron size length scales studied here.

Furthermore, universal conductance fluctuations previously observed in $\mathrm{HgTe} / \mathrm{CdTe}$ quantum wells are significantly suppressed in our experiments, indicating a more
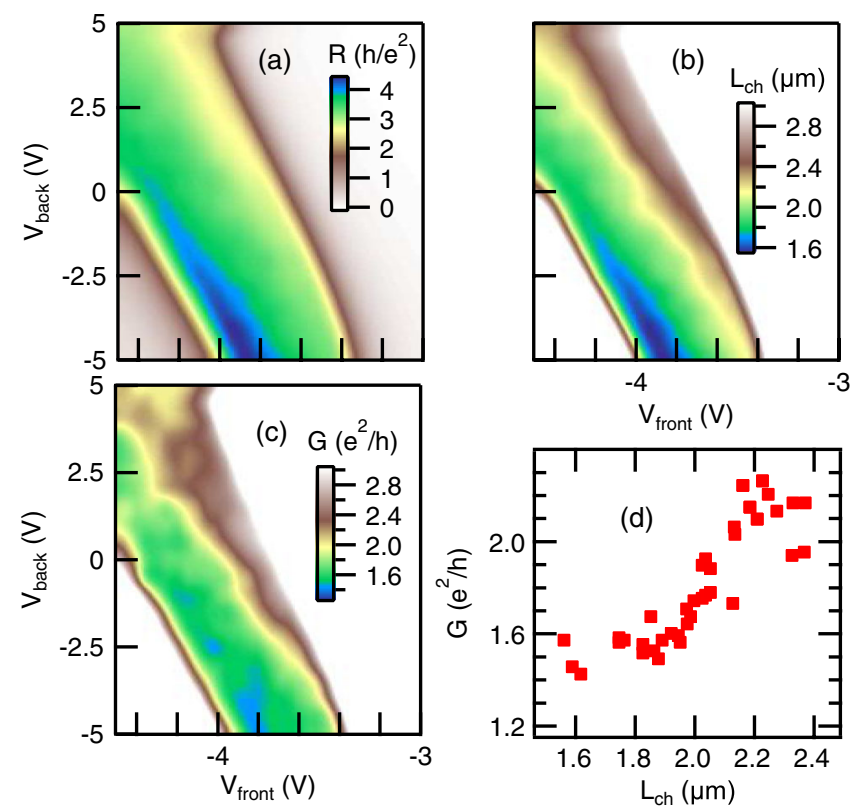

FIG. 3 (color online). Panel (a) shows $R_{1-3,5-4}$ with $L_{5-4}=$ $20.5 \mu \mathrm{m}$ in the self-averaged regime, while panel (b) shows the extracted characteristic length of the ballistic edge transport $L_{\mathrm{ch}} \sim\left(h / 3 e^{2}\right)\left(L_{5-4} / R_{1-3,5-4}\right)$, as function of $V_{\text {front }}$ and $V_{\text {back }}$, at $B=0 \mathrm{~T}, T=20 \mathrm{mK}$. Similarly, panel (c) shows the conductance $G_{1-3 \& 4,6-5}$, with $L_{6-5}=1.8 \mu \mathrm{m}$ in the ballistic regime. Suppression of the edge conductance is correlated with the reduction of $L_{\mathrm{ch}}$ as shown in panel (d) and occurs for more negative $V_{\text {front }}$ and $V_{\text {back }}$. 
robust helical state. Nevertheless, Fig. 2 shows that the resistance values in the topological gap are slightly larger than $h / 2 e^{2}$ for more negative gate biases, suggesting that $L_{\mathrm{ch}}$ may be reduced with a more negative gate bias. As a result, the self-averaged regime is investigated by measuring the voltage between macroscopically separated leads 5 and $4, L_{5-4}=20.5 \mu \mathrm{m}$, while the current is passed from leads 1 to 3 . The corresponding resistance map $R_{1-3,5-4}$ at $B=0 \mathrm{~T}$ is shown in Fig. 3(a), and is seen to increase for more negative $V_{\text {front }}$ and $V_{\text {back }}$. The characteristic length is extracted through the relation, $L_{\mathrm{ch}} \sim\left(h / 3 e^{2}\right)\left(L_{5-4} / R_{1-3,5-4}\right)$, and is plotted in Fig. 3(b). At fixed $V_{\text {front }}\left(V_{\text {back }}\right), L_{\text {ch }}$ decreases for reduced $V_{\text {back }}$ $\left(V_{\text {front }}\right)$, indicating stronger backscattering processes. This result suggests that the dominant backscattering process may be driven by the presence of mobile charged species of a negative sign in the AlSb barriers or dielectric layer, which are pushed closer to the quantum well for more negative gate biases, or similarly filling of charge traps in close proximity to the well. The gate bias dependence of $L_{\mathrm{ch}}$ explains the concomitant edge conductance suppression for mesoscopically separated probes, shown in Fig. 2, and replotted in Fig. 3(c) as a conductance map $G_{1-3 \& 4,6-5}$. Figure 3(d) shows a sample of conductance values from Fig 3(c) plotted versus $L_{\mathrm{ch}}$ obtained at the same gate biases in Fig. 3(b). For $L_{\mathrm{ch}}>2.2 \mu \mathrm{m}$ conductance plateaus at approximately $2 e^{2} / h$, while for smaller $L_{\mathrm{ch}}$, edge conductance monotonically decreases. The separation $L_{6-5}=1.8 \mu \mathrm{m}$ and thus the edge transport is in the ballistic regime only for more positive gate biases. In consequence, in this experiment evolution of conductance from ballistic to the self-averaged disordered regime is observed as the gate biases are made more negative.

Finally, we briefly discuss the magnetic field and temperature dependence of both ballistic and self-averaged transport shown in Figs. 4(a) and 4(b), respectively. In both cases, we observe no significant change in resistance values for temperatures between $20 \mathrm{mK}$ and $1 \mathrm{~K}$, and magnetic fields up to $1 \mathrm{~T}$ along all principal directions. While this magnetic field dependence is in seeming contradiction with the TRS protected state $[5,6]$, we argue that the helical edge state in $\mathrm{InAs} / \mathrm{GaSb}$ must be more robust to the magnetic fields due to the low Fermi velocity of the HL and a particularly small orbital $g$ factor. The Fermi velocity is estimated from the crossing vector and the gap size to be $v_{F} \sim 1.8 \times 10^{4} \mathrm{~m} / \mathrm{s}$, giving an unusually small orbital $g$ factor $g \sim 0.47$ [22]. In this case, magnetic fields of even up to $10 \mathrm{~T}$ would open up a gap in the spectrum of only $\lesssim 0.3 \mathrm{meV}$, which is comparable to the level broadening [20] and, hence, would not be apparent in transport measurements. Besides the low effective $g$ factor, the weak magnetic field dependence may be explained via the fact that the Dirac point of the edge states may actually be "hidden" between the two hole band maxima [unlike presented in the inset of Fig. 1(c)] [23]. On the other hand,
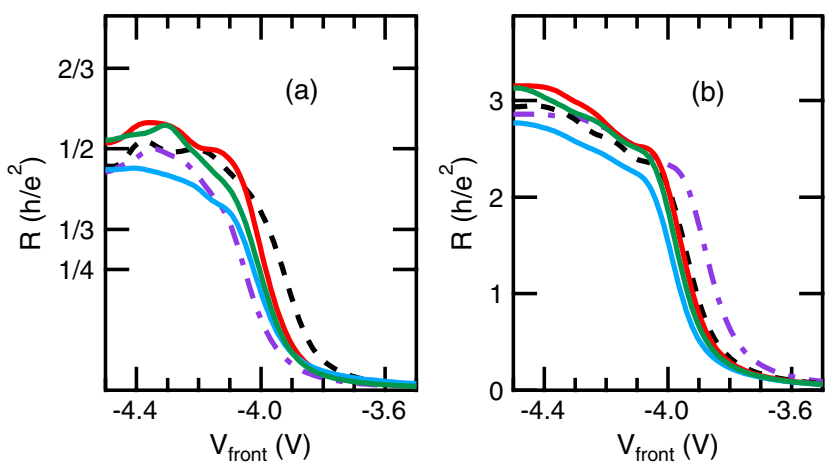

FIG. 4 (color online). Panel (a) and (b) show $R_{1-3 \& 4,6-5}$ and $R_{1-3,5-4}$ which are in ballistic and self-averaged regimes, respectively, as a function of $V_{\text {front }}$ and fixed $V_{\text {back }}=5 \mathrm{~V}$, at $T=20 \mathrm{mK}, B=1 \mathrm{~T}$ (full lines), along $x$ (in red), $y$ (in blue), and $z$ (in green) directions, and $B=0 \mathrm{~T}$ (dashed lines), $T=20 \mathrm{mK}$ (in black), and $T=1 \mathrm{~K}$ (in violet).

the absence of strong temperature dependence in our measurements indicates that elastic single particle backscattering, i.e., spin-flip scattering, dominates over single particle inelastic and multiparticle processes, which exhibit strong power laws [14]. In fact, helical edge states are expected to be robust to normal dephasing [18] as brought on by electron-electron and electron-phonon interactions [24], while particularly sensitive to spin-flip dephasing processes [18]. In addition, the energy scale of the inelastic scattering such as from nearby charge puddles [15] is $\hbar v_{F} / l_{\text {inelastic }} \lesssim 70 \mathrm{mK}$, where the inelastic scattering length $l_{\text {inelastic }}$ is several $\mu \mathrm{m}$ [20], and, hence, a weak temperature dependence observed here may not be all that surprising. Finally, a low Fermi velocity would indicate that correlations may be important in the edge physics of the TI InAs/GaSb [16], albeit the presence of the electrostatic gates leads to significant screening.

The work at IBM has been supported by the DARPA MESO Project No. N66001-11-1-4105. L. D. and R. R. D. gratefully acknowledge support from NSF Grant No. DMR-1207562 and Welch Foundation Grant No. C1682. We thank Dmitry Pikulin and Emil Prodan for fruitful discussions as well as Noel Arellano and Brian Hughes for assistance in sample fabrication.

[1] C. L. Kane and E. J. Mele, Phys. Rev. Lett. 95, 226801 (2005).

[2] B. A. Bernevig, T. L. Hughes, and S.-C. Zhang, Science 314, 1757 (2006).

[3] M. Z. Hasan and C. L. Kane, Rev. Mod. Phys. 82, 3045 (2010).

[4] X.-L. Qi and S.-C. Zhang, Rev. Mod. Phys. 83, 1057 (2011).

[5] C. Wu, B. A. Bernevig, and S.-C. Zhang, Phys. Rev. Lett. 96, 106401 (2006).

[6] C. Xu and J. E. Moore, Phys. Rev. B 73, 045322 (2006). 
[7] M. Konig, S. Wiedmann, C. Brune, A. Roth, H. Buhmann, L. W. Molenkamp, X.-L. Qi, and S.-C. Zhang, Science 318, 766 (2007).

[8] A. Roth, C. Brüne, H. Buhmann, L. W. Molenkamp, J. Maciejko, X.-L. Qi, and S.-C. Zhang, Science 325, 294 (2009).

[9] L. Du, I. Knez, G. Sullivan, and R. R. Du, arXiv:1306.1925.

[10] C. Liu, T. L. Hughes, X.-L. Qi, K. Wang, and S.-C. Zhang, Phys. Rev. Lett. 100, 236601 (2008).

[11] I. Knez, R.-R. Du, and G. Sullivan, Phys. Rev. Lett. 107, 136603 (2011).

[12] G. M. Gusev, Z. D. Kvon, O. A. Shegai, N. N. Mikhailov, S. A. Dvoretsky, and J. C. Portal, Phys. Rev. B 84, 121302(R) (2011).

[13] T. L. Schmidt, S. Rachel, F. Von Oppen, and L. I. Glazman, Phys. Rev. Lett. 108, 156402 (2012).

[14] N. Lezmy, Y. Oreg, and M. Berkooz, Phys. Rev. B 85, 235304 (2012).
[15] J. I. Väyrynen, M. Goldstein, and L. I. Glazman, Phys. Rev. Lett. 110, 216402 (2013).

[16] J. Maciejko, C. Liu, Y. Oreg, X.-L. Qi, C. Wu, and S.-C. Zhang, Phys. Rev. Lett. 102, 256803 (2009).

[17] Y. Tanaka, A. Furusaki, and K. A. Matveev, Phys. Rev. Lett. 106, 236402 (2011).

[18] H. Jiang, S. Cheng, Q. F. Sun, and X. C. Xie, Phys. Rev. Lett. 103, 036803 (2009).

[19] Y. Naveh and B. Laikhtman, Europhys. Lett. 55, 545 (2001).

[20] I. Knez, R. R. Du, and G. Sullivan, Phys. Rev. B 81, 201301 (R) (2010).

[21] I. Knez, R.-R. Du, and G. Sullivan, Phys. Rev. Lett. 109, 186603 (2012).

[22] G. Tkachov and E. M. Hankiewicz, Phys. Rev. B 83, 155412 (2011).

[23] E. Prodan, private communications.

[24] J. C. Budich, F. Dolcini, P. Recher, and B. Trauzettel, Phys. Rev. Lett. 108, 086602 (2012). 